\title{
The Learning Effectiveness of Nurses Participating in a Simulated Management of Fall Risk Factor Assessment
}

\author{
Chih-Ying $\mathrm{Tu}^{1}$, Sheng-Hong $\operatorname{Lin}^{2,3 *}$ \\ ${ }^{1}$ Department of Nursing, Tri-Service General Hospital, Taipei, Taiwan \\ ${ }^{2}$ Division of Allergy, Immunology and Rheumatology, Department of Internal Medicine, Shuang Ho Hospital, Taipei \\ Medical University \\ ${ }^{3}$ Division of Allergy, Immunology and Rheumatology, Department of Internal Medicine, School of Medicine, College \\ of Medicine, Taipei Medical University, Taipei, Talwan \\ * Sheng-Hong Lin: shlin0317@gmail.com
}

\begin{abstract}
Background: Fall injuries are common causes of dangerous inpatient conditions and should be prevented in neurosurgery inpatient wards. An adequate survey and evaluation of risk factors for falls is therefore crucial for the development of prevention and intervention strategies in inpatient care. Purpose: To investigate the learning effectiveness of a simulated management system for improving nurses' fall risk assessments and management. Method: A total of 12 nurses at a hospital in one health system in northern Taiwan who had ever used a "fall risk factor assessment" tool for patients undergoing neurosurgery were enrolled in this quasi-experimental study. A single group with a pre-test and post-test (6 weeks after intervention) approach was constructed. For our simulated model of a fall risk factor assessment, we used a strategy of teaching/practice on the simulated model. The outcomes were evaluated with a "questionnaire for fall risk factor assessment". Results: The within-subject effects indicated notable improvement in knowledge of fall risk factors after our strategy of teaching/practice with simulated model training was implemented. The mean scores on knowledge of fall risk factors were 3.25 and 9.13 on the pre-test and post-test, respectively. We found a remarkable improvement in the post-test scores compared with the pre-test results among participants who implemented our training strategies. Conclusion: The simulated method for fall risk factor assessment with a teaching/practice training process demonstrated a significant and favorable benefit on learning outcomes, especially as a new staff training program. We recommended incorporating a simulated system with teaching/practice into clinical nursing education and training strategies to improve training courses.
\end{abstract}

Keywords: fall risk factor, simulation, nurse.

\section{INTRODUCTION}

According to statistics from the World Health Organization (WHO), approximately 640,000 people die due to falls every year, and most of them are older than 65 years old [1]. In the United States, $50 \%$ of hospitalized patients are at risk of falling, and $30-50 \%$ of falls result in a series outcomes ranging from mild injury to death. In addition to causing physical damage, prolonged hospital stays, and increased cost of medical resources, falls also are associated with many negative emotions and impacts on patients and their families, including fear of falling, worry, depression, guilt, remorse, activity restriction, or decreased quality of life [2]. Once an inpatient falls, the hospital stay may be prolonged by 6-12 days, and the medical expenses may increase by approximately $\$ 13,316.60$ [3], indicating the importance of fall prevention.
Falls are not caused by a single factor; the reasons are complex and mutually influential. The risk factors for inpatient falls depend on the particular patient's condition (internal factor) and hospital environment (external factor). Internal factors refer to each patient's physicalor health-related factors and include age, frequent urination, delirium, visual inaccuracy, unstable gait, multiple medication prescriptions, previous fall history, personal activity level, limb weakness, and consciousness status. External factors include uneven surfaces, wet environment, lack of assistance from others and non-use of auxiliary tools [4].

One-third of all falls can be prevented by using an effective assessment tool including education about multiple risk factors for fall prevention for medical teams, patients, or caregivers and by providing individualized and diversified fall prevention measures in 
combination with cross-team resources [5]. In the education and training of health practitioners, it is necessary to strengthen the use of risk assessment tools and the vigilance of maintaining a safe environment, which can improve fall prevention and facilitate immediate identification of the risk factors for patient falls to remove them [3].

Simulated situational learning involves construction of a real situation or environment for learning. The situation needs to be real and similar to clinic conditions. In the beginning of the learning course, a schedule and learning objectives should be introduced. After providing the basic knowledge innovation, a simulated situational learning course can commence to allow learners to develop long-term memories of knowledge and technology and to improve teaching effectiveness [6]. In general, simulated situational teaching utilizes the standard patients, part functional practice devices, simulated electronic patient trainers, role playing, peer learning, and virtual reality. Simulated function trainers are mainly utilized to simulate human partial body functions, providing technical drills or execution of process operation, to improve learners' clinical care ability [7]. In the process of situational simulation learning, learners can gain emotional, cognitive, and technical experience, improve their comprehension, evaluation, analysis, decision-making, practical and coping abilities, and meanwhile increase their learning motivation, sense of security, confidence, and satisfaction $[6,8]$.

Increased accuracy of risk assessment for falls by nursing practitioners can improve the quality of health care and ameliorate patient injury. The effectiveness of fall assessment education for junior staff remains lacking. Simulated situational training is effective education measure. Therefore, our study was constructed to investigate the learning effectiveness of a simulated management system to improve nurses' fall risk assessments and management.

\section{METHODS}

In our study, a single group with a pre-test and posttest research design was adopted. The participants in our study included nurse practitioners who served in the neurosurgery ward of a medical center in Northern Taiwan. After obtaining consent, a total of 12 nurse practitioners were included and participated in a 30minute simulated situational learning course. The course content included fall assessment and fall-related management topics. The pre-test was taken before the course, and the post-test was taken six weeks after participation in the simulated situational learning course.
The questionnaire consisted of three parts: (1) personal background information, including gender, age, education, nursing period, any experience with nursing patients who had encountered fall events, and any participation in fall assessment courses and (2) a fall assessment knowledge, attitude, and behavior questionnaire. In the latter part of the questionnaire, ten questions about fall assessment knowledge were rated as "true" and "false", and ten questions each about attitude and behavior (about the fall management process) were scored on a 4-point Likert scale (1 point indicates strongly disagree, and 4 points indicates strongly agree). A total of 30 questions were included on the pre-test and post-test. The validity of the questionnaire used in this study was based on expert content validity, and the internal consistency assessment with Cronbach's $\alpha$ was 0.92 after experts' opinions were provided to modify the questionnaire.

\section{RESULTS}

Twelve nurses, with an average age of $27.42 \pm 9.1$ years and an average working experience period of $5.63 \pm 7.48$ years, participated in our study. Most of them had 2-5 years of working experience $(58.33 \%)$. Among them, 10 had experience in nursing patients with fall events $(83.33 \%)$, and only 5 had participated in education courses related to fall assessments $(58.33 \%)$.

In terms of knowledge, the average scores on the pretest and post-test were 2.25 and 9.13 , respectively. In terms of attitude, the average scores on the pre-test and post-test were 6.92 and 7.75 , respectively. In terms of behavior (fall handling), the mean scores on the pre-test

Table 1. Learning effectiveness of a simulated management

\begin{tabular}{|l|c|c|}
\hline Item & $\begin{array}{c}\text { Pre-test } \\
\text { (mean score) }\end{array}$ & $\begin{array}{c}\text { Post-test } \\
\text { (mean score) }\end{array}$ \\
\hline Knowledge & 2.25 & 9.13 \\
\hline Attitude & 6.92 & 7.75 \\
\hline $\begin{array}{l}\text { Behavior } \\
\text { (fall handling) }\end{array}$ & 6.83 & 7.25 \\
\hline
\end{tabular}

and post-test were 6.83 and 7.25, respectively.

\section{DISCUSSION}

In our study, the within-subject effects indicated notable improvement in knowledge in the fall risk factors assessment by receiving our strategies of teaching/practice in the simulated situational model training. The mean scores for knowledge on the fall risk factor assessment were 2.25 and 9.13 on the pre-test and post-test, respectively. We found a remarkable 
improvement on the post-test scores compared with the pre-test scores in participants educated with our training strategies.

The main purposes of situational simulation learning include the following: procedural training, evaluation of individual responses in a crisis, evaluation of equipment, task analysis, and group training. Complete clinical simulation scenarios allow clinical health caregivers to practice nursing techniques in a safe, controlled, realistic setting using clinical products. This enables learners to improve their technical ability and critical thinking, gain confidence, have increased satisfaction with their learning experience, and receive enhanced feedback and reflection after situational simulation training [9].

In our study, more than half of the 12 participant nurses had not received education about fall assessments. This finding highlights that clinical nursing practitioners had not obtained education about fall evaluation. Notably, hospital managers should strengthen continuing education about fall assessment because a cognitive deficiency among nursing practitioners regarding assessing falls will influence the screening and prevention measures for patients at high risk of falls. Through the simulated situational training provided in our study, the fall assessment knowledge score after 6 weeks remarkably improved from 2.25 to 9.13 , This is consistent with literature showing that health caregivers increase their alertness to falls through training [10].

We recommended incorporating a simulated situational system with teaching/practice into clinical nursing education and training strategies to yield better training outcomes.

Due to the restrictions of the manpower, time, and administrative support, a control group was not recruited for our study. The number of participants should be expanded in future studies. For the more accurate and validated effects, a case-control method could be used in subsequent studies. The post-test time can also be extended in the future to track the long-term learning outcomes and increase the power of the effects found in our study. A second limitation is the limited number of participants owing to the need to coordinate the times of the instructors and trainees. To address this, the teaching part can be replaced by self-learning tools or e-learning methods.

\section{CONCLUSION}

A simulated situational training course can improve the training outcomes of fall assessments among nursing practitioners to help prevent inpatient injuries from fall accidents.

\section{AUTHORS' CONTRIBUTIONS}

C.Y. Tu developed the theoretical formalism, performed the analytic calculations and performed the numerical simulations. Both C.Y. Tu and S.H. Lin contributed to the final version of the manuscript.

\section{ACKNOWLEDGMENTS}

We thank all the nursing participants in our study for assistance with our research.

\section{REFERENCES}

[1] World Health Organization. (2018). Falls. World Health Organization,. Retrieved March 11, 2021 from https://www.who.int/news-room/factsheets/detail/falls

[2] D. Avanecean, D. Calliste, T. Contreras, et al., Effectiveness of patient-centered interventions on falls in the acute care setting: a quantitative systematic review protocol. JBI Database System Rev Implement Rep, 2017, pp. 55-65. DOI: https://doi.org/ 10.11124/JBISRIR-2016-002981.

[3] Y.L. Zhao, M. Bott, J. He, et al., Evidence on Fall and Injurious Fall Prevention Interventions in Acute Care Hospitals. J Nurs Adm, 2019, pp. 86-92. DOI: https://doi.org/ 10.1097/NNA.0000000000000715.

[4] C.P. Remor, C.B. Cruz,S. Urbanetto Jde, [Analysis of fall risk factors in adults within the first 48 hours of hospitalization]. Rev Gaucha Enferm, 2014, pp. 28-34. DOI: https://doi.org/ 10.1590/19831447.2014.04.50716.

[5] K.I. Kim, H.K. Jung, C.O. Kim, et al., Evidencebased guidelines for fall prevention in Korea. Korean J Intern Med, 2017, pp. 199-210. DOI: https://doi.org/ 10.3904/kjim.2016.218.

[6] L.B. Nilson, Teaching at its best: A research-based resource for college instructors. 2016: John Wiley \& Sons.

[7] J. Norman, Systematic review of the literature on simulation in nursing education. ABNF J, 2012, pp. 24-28.

[8] D. A. Cook, R. Hatala, R. Brydges, et al., Technology-enhanced simulation for health professions education: a systematic review and meta-analysis. JAMA, 2011, pp. 978-988. DOI: https://doi.org/ 10.1001/jama.2011.1234.

[9] C. Bailey, J. Johnson-Russell,A. Lupien, Highfidelity patient simulation. Innovative teaching 
strategies in nursing and related health professions, 2011, pp. 207-226.

[10] C. Weiss, E. Holland, G. Cable, et al., An intervention to prevent falls in the elderly: a time series quasiexperiment. Clin Excell Nurse Pract, 2002, pp. 55-60. 